\title{
Lateral resolution in focused electron beam-induced deposition: scaling laws for pulsed and static exposure
}

\author{
Aleksandra Szkudlarek • Wojciech Szmyt • \\ Czesław Kapusta $\cdot$ Ivo Utke
}

Received: 4 April 2014/ Accepted: 27 August 2014/Published online: 18 September 2014

(C) The Author(s) 2014. This article is published with open access at Springerlink.com

\begin{abstract}
In this work, we review the single-adsorbate time-dependent continuum model for focused electron beam-induced deposition (FEBID). The differential equation for the adsorption rate will be expressed by dimensionless parameters describing the contributions of adsorption, desorption, dissociation, and the surface diffusion of the precursor adsorbates. The contributions are individually presented in order to elucidate their influence during variations in the electron beam exposure time. The findings are condensed into three new scaling laws for pulsed exposure FEBID (or FEB-induced etching) relating the lateral resolution of deposits or etch pits to surface diffusion and electron beam exposure dwell time for a given adsorbate depletion state.
\end{abstract}

\section{Introduction}

Focused electron beam-induced deposition (FEBID) is a maskless direct-write nanolithography technique, in which

Electronic supplementary material The online version of this article (doi:10.1007/s00339-014-8751-2) contains supplementary material, which is available to authorized users.

A. Szkudlarek ( $\square) \cdot$ I. Utke

Laboratory for Mechanics of Materials and Nanostructures,

Empa, Feuerwerkerstrasse 39, 3602 Thun, Switzerland

e-mail: aleszkud@agh.edu.pl

I. Utke

e-mail: ivo.utke@empa.ch

A. Szkudlarek · W. Szmyt - C. Kapusta

Department of Solid State Physics, Faculty of Physics and Applied Computer Science, AGH University of Science and Technology, al. A. Mickiewicza 30, 30-059 Kraków, Poland the precursor gas molecules are directly delivered into the electron microscope chamber, where they are adsorbed onto the substrate surface and dissociated via the interaction with the focused electron beam [1]. In such a way, local functional nanostructures can be formed without multistep processing, which is necessary in common resistbased electron beam lithography. Among the functional materials that have been fabricated using FEBID are ferromagnetic wires [2-4], metallic [5], and graphitic material [6] for low-resistance nanocontacts, as well as granular wires for strain sensors [7], magnetic sensors [8], gas sensors [9], and material with photonic/plasmonic functionality [10-13].

Although the above-sketched concept of FEBID seems to be simple, the final product depends on the three types of interactions: electron beam-substrate, electron beamadsorbates, and adsorbates-substrates. The interaction between electron beam and adsorbates involves already several parameters related to the adsorbate surface kinetics and their dissociation efficiency by the electron beam. As a result, already for the simple case of stationary electron beam (spot) exposures, various types of deposit shape profiles can be obtained, see (Fig. 1). These shape profiles were well described by the FEBID continuum model [1416]. When the electron beam-dissociated adsorbates are instantaneously replenished by new adsorbates or incoming molecules, the deposit shape corresponds to the Gaussian profile of the beam and the process is carried out in the electron-limited regime (Fig. 1a). Should the adsorbates be depleted inside the irradiated area, the surface diffusion of adsorbates from the surrounding non-irradiated area starts to play a considerable role. A characteristic indent shape is often obtained for the diffusion-enhanced regime (Fig. 1b) as adsorbates diffusing to the center of the irradiated area are dissociated at its rim by the electron beam tails. If 
Fig. 1 Deposit shapes and process regimes for stationary single-spot exposures with a Gaussian profile electron beam. The generic shapes correspond to the process regimes: a Gaussian shape for electronlimited regime (also known as reaction rate-limited regime), b Indent shape for diffusionenhanced regime, c Flattop shape for adsorbate-limited regime (also known as mass transport-limited regime), d Rounded shape for a mixed diffusion-enhanced/adsorbatelimited regime

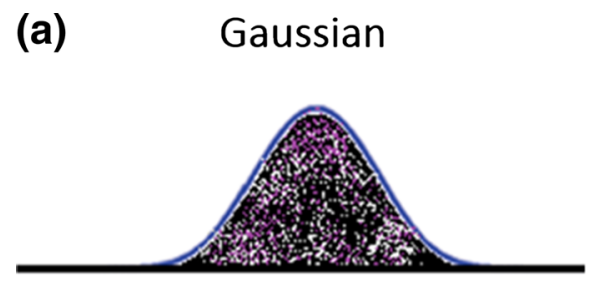

Electron-limited

(Reaction-rate-limited)

(c)

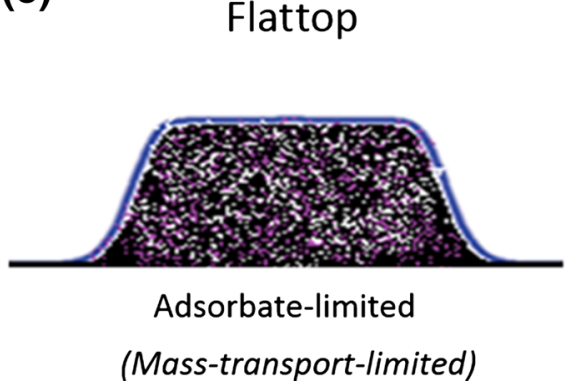

(b) Indent

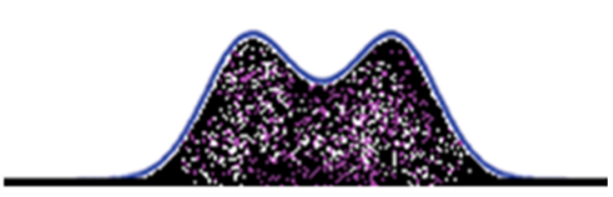

Diffusion-enhanced

(d)

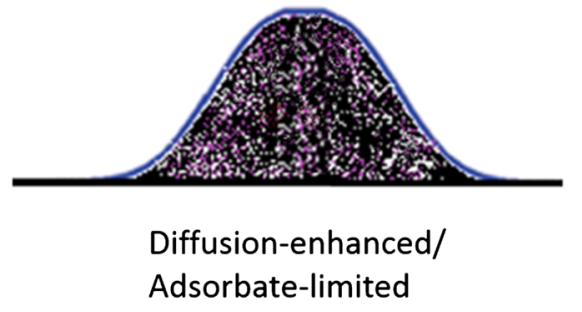

surface diffusion is strong, the adsorbates can reach the center and a round-shaped deposit is obtained (Fig. 1d). In the last case, in the adsorbate-limited regime when the depletion rate is larger than the adsorbate replenishment and when the surface diffusion is weak, the flattop deposit shape is observed (Fig. 1c).

\section{Continuum model}

The continuum model for stationary FEBID was previously described in detail in $[15,16]$. Here, we propose a general approach, referring to the time-dependent version of the adsorption rate equation with a set of two independent parameters, which describe the adsorbate surface diffusion and the irradiative depletion. This methodology can be applied to focused electron beam-induced deposition and etching to obtain the shapes of the deposit or the respective etch pit. The solution of the stationary-state adsorption rate equation using this methodology has already been discussed in [16]. Several precursor-specific approaches were reported in the literature: the fundamental interactions between the beam and adsorbate in pulsed FEBID are discussed in [17], taking $\mathrm{W}(\mathrm{CO})_{6}$ as a gas precursor. Experimental etch shapes using $\mathrm{XeF}_{2}$ as a precursor gas have been correlated with the process parameters in [18, 19]. A transition between simultaneous etching and deposition of the contamination deposit was discussed in [20]. The continuum model, which explains this effect including two types of adsorbates species_-one etching and another one forming a deposit-was developed in [21]. Simultaneous co-adsorption of two precursor gases for deposition without taking surface diffusion into account was studied in [22, 23]. Specific phenomena which may appear during the FEBID process, like chemisorption or electron-stimulated desorption of adsorbates, were proposed in [24] and [25], respectively.

In the following, we want to discuss more generally the evolution of the individual contributions of adsorption, desorption, irradiative depletion, and surface diffusion with electron beam exposure time in order to derive scaling laws for the lateral resolution in deposition and etching with focused electron beams.

\section{Concentration of adsorbates-surface coverage}

The local number $n$ of adsorbates at a given area on the surface can change due to the four following processes, which are schematically presented in Fig. 2:

- adsorption, which is proportional to the impinging rate of precursor gas molecules $J$ and to the number of available places $\left(1-\frac{n}{n_{0}}\right)$ with $n_{0}$ the adsorbates concentration of a complete monolayer; as there is no condensation process occurring, $n_{0}$ represents the maximum value for $n$

- desorption, where the physisorbed molecules have a given average residence time $\tau$, after that they spontaneously desorb thermally from the surface

- dissociation, the rate of which is proportional to the electron flux $f(r)$ and the number of available adsorbates 


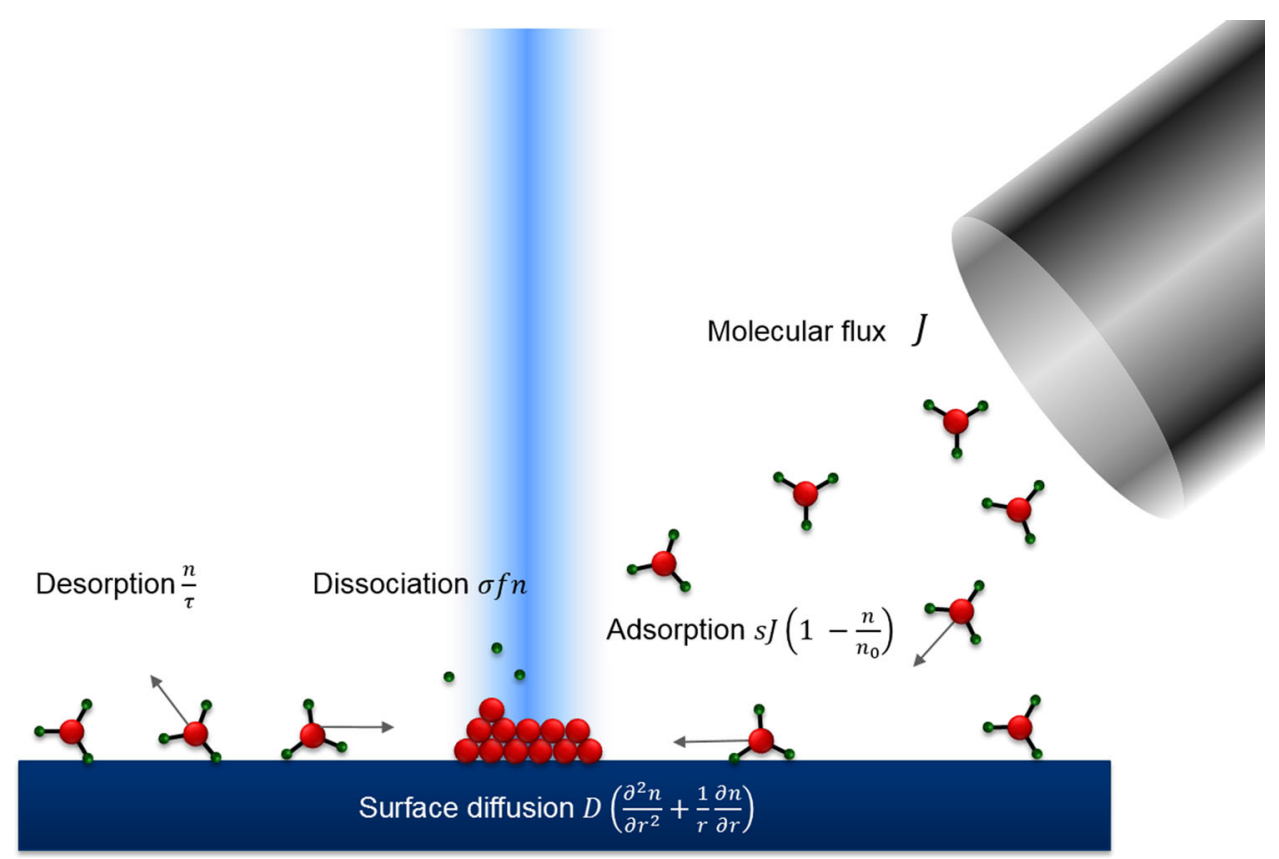

Fig. 2 Physicochemical mechanisms in FEBID process: (1) adsorption-proportional to the sticking probability, impinging molecular flux, and non-occupied adsorption sites; (2) thermal spontaneous desorption-inversely proportional to the average residence time characteristic for a given adsorbate; (3) electron-induced dissociation-depending on the electron flux, number of adsorbates, and the efficiency of the electron-induced dissociation reaction described by the net dissociation cross section; (4) surface diffusionbeing proportional to the divergence of the gradient of adsorbates and the surface diffusion constant as a proportionality factor
Fig. 3 Variables used in the continuum model. The Gaussian electron beam is described by the electron flux at the center of the beam $f_{0}$ and the full-width at half-maximum $\mathrm{FWHM}_{\mathrm{B}}$.

Outside the irradiated area, the adsorbate concentration is $n_{\text {out }}$ and the adsorbate surface diffusion path is $\rho_{\text {out }}$. Based on these variables, the two dimensionless surface kinetic parameters can be defined: irradiative adsorbate depletion $\tilde{\tau}$ and surface diffusion replenishment $\tilde{\rho}_{\text {out }}$, see also text

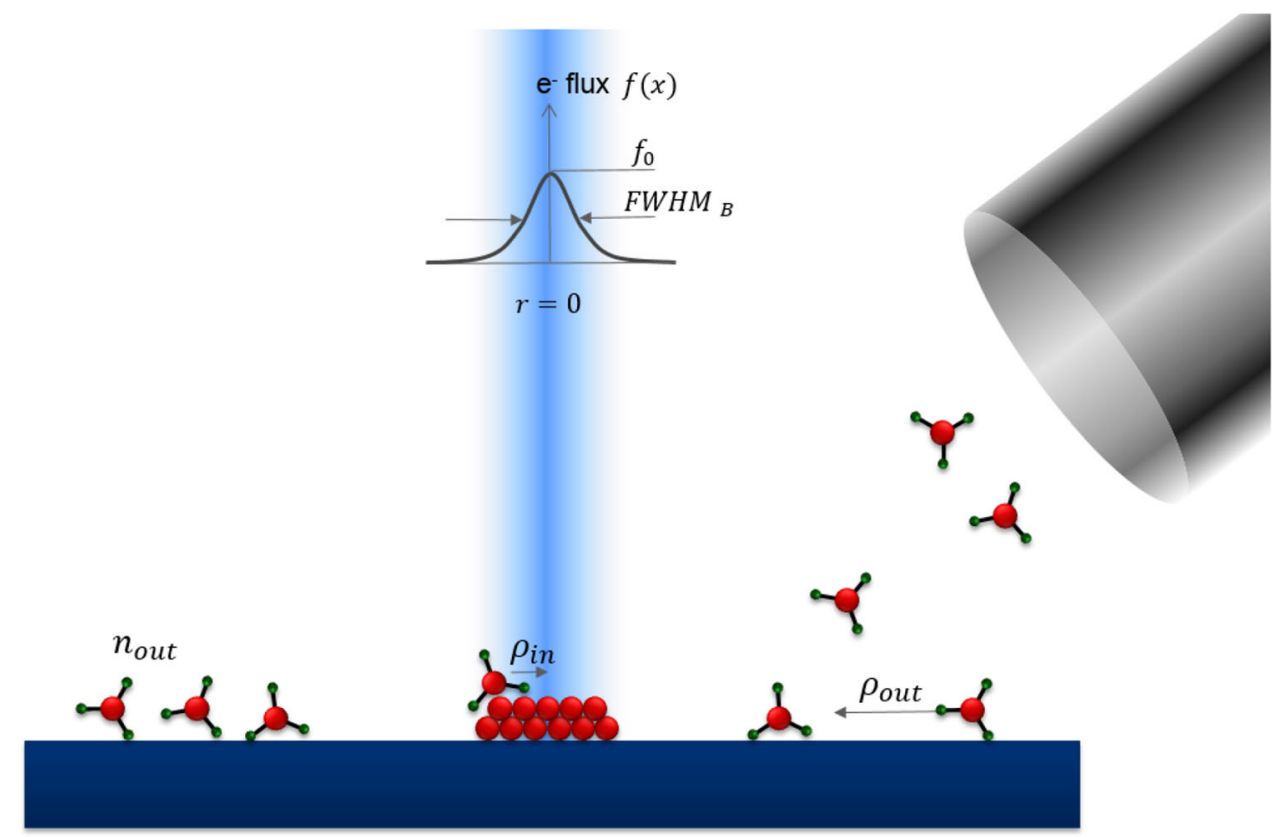

with a constant $\sigma=\sigma\left(E_{B}\right)$ called a net dissociation cross section, depending on the beam energy $E_{\mathrm{B}}$ and the chemical composition of the adsorbate

- surface diffusion, which is proportional to the adsorbate diffusivity described by the surface diffusion coefficient $D$ and the Laplacian of adsorbate concentration $\nabla^{2} n=\frac{\partial^{2} n}{\partial x^{2}}+\frac{\partial^{2} n}{\partial y^{2}}$ with $\nabla$ being the Nabla operator. This expression applies to non-interacting adsorbates and represents Fick's law of diffusion.

The adsorption rate equation describing the change in the local concentration of adsorbates is 


$$
\frac{\partial n}{\partial t}=\overbrace{s J\left(1-\frac{n}{n_{0}}\right)}^{\text {adsorption }} \underbrace{-\frac{n}{\tau}}_{\text {desorption }} \overbrace{-\sigma f n}^{\text {dissociation }} \underbrace{+D\left(\frac{\partial^{2} n}{\partial x^{2}}+\frac{\partial^{2} n}{\partial y^{2}}\right)}_{\text {diffusion }},
$$

where $n(x, y, t)$ - concentration of adsorbates, $D$-surface diffusion coefficient, $\tau$-residence time of adsorbates, $J$ molecular flux, $s$-sticking probability, and $n_{0}$-maximum concentration of adsorbates in one monolayer.

Equation 1 can be rewritten using an effective residence time and taking into account the rotational symmetry of the problem (Fig. 3)

$\frac{\partial n}{\partial t}=s J \overbrace{-\frac{n}{\tau_{\text {out }}}}^{\text {eff.desorption }}-\sigma f n+D\left(\frac{\partial^{2} n}{\partial r^{2}}+\frac{1}{r} \frac{\partial n}{\partial r}\right)$.

The effective residence time, $\tau_{\text {out }}:=\left(\frac{1}{\tau}+\frac{s J}{n_{0}}\right)^{-1}$, combines the instant desorption from the surface if the adsorption site is already occupied by an adsorbate and the spontaneous thermal desorption after an average residence time $\tau$.

\section{Parameterization of surface diffusion, irradiative adsorbate depletion}

The dimensionless parameter of irradiative depletion of adsorbates $\tilde{\tau}$ is the ratio of intact adsorbates outside the irradiated area with respect to those staying intact at the center of the beam, if there is no contribution of surface diffusion replenishment. It is defined as:

$\tilde{\tau}=1+\sigma f_{0} \tau_{\text {out }}$

It combines the net dissociation cross section $\sigma$, effective residence time of adsorbates $\tau_{\text {out }}$, and the electron flux at the center of the beam $f_{0}$.

A value close to one means that the electron-limited regime prevails, as the adsorbate concentration at the center of the beam will be close to the adsorbate concentration established by adsorption desorption processes at equilibrium state outside of the irradiated area.

The surface diffusion can be characterized by the following parameter, defined as the ratio between the effective diffusion length and the $\mathrm{FWHM}_{\mathrm{B}}$ :

$\tilde{\rho}_{\text {out }}:=\frac{2 \sqrt{D \tau_{\text {out }}}}{\text { FWHM }_{\mathrm{B}}}=\frac{2 \rho_{\text {out }}}{\text { FWHM }_{\mathrm{B}}}$.

In the case of a Gaussian profile of the electron beam, a small value of surface diffusion replenishment $\tilde{\rho}_{\text {out }} \ll 0.1$ means that the surface diffusion contribution can be neglected. As a result for a large depletion value $\tilde{\tau} \gg 1$, a flat deposit shape is obtained being characteristic for the adsorbate-limited regime.
In order to reduce the adsorbate rate equation to a dimensionless form, the dimensionless variables are introduced $\bar{n}=n / s J \tau_{\text {out }}, \bar{f}=\frac{f}{f_{0}}, \bar{t}=\frac{t}{\tau_{\text {out }}}, \bar{r}=\frac{2 r}{\text { FWHMB }}$ :

$$
\begin{gathered}
\frac{\partial \bar{n}}{\partial \bar{t}}=1-\tilde{k}(\bar{r}) \bar{n}+\tilde{\rho}_{\text {out }}^{2}\left(\frac{\partial^{2} \bar{n}}{\partial \bar{r}^{2}}+\frac{1}{\bar{r}} \frac{\partial \bar{n}}{\partial \bar{r}}\right), \\
\lim _{\bar{r} \rightarrow 0} \frac{\partial \bar{n}}{\partial \bar{r}}=0,(\text { B.C.1) } \\
\lim _{\bar{r} \rightarrow \infty} \bar{n}(\bar{r})=1,(\text { B.C. } 2) \\
\bar{n}(\bar{t}=0)=1 .(\text { I.C. })
\end{gathered}
$$

where $\tilde{k}=\tilde{k}(\bar{r})=1+(\tilde{\tau}-1) 2^{-\bar{r}^{2}}$ is a term combining adsorbate desorption and dissociation, and it is equal to $\tilde{\tau}$ at the center of the beam. The Eq. 1B can be solved numerically with the surface diffusion term or analytically when the surface diffusion term is neglected.

\section{Adsorbates concentration in pulsed FEBID without surface diffusion}

In the case of negligible surface diffusion contribution, the adsorbate concentration, derived from Eq. $1 \mathrm{~B}$ at a given normalized time $\left(\bar{t}=t / \tau_{\text {out }}\right)$, is expressed as

$$
\bar{n}(\bar{t})= \begin{cases}1 / \tilde{k}+(1-1 / \tilde{k}) \exp (-\tilde{k} \bar{t}), & \bar{t} \leq \bar{t}_{\mathrm{D}} \\ (1-1 / \tilde{k})\left(\exp \left(-\tilde{k} \bar{t}_{\mathrm{D}}\right)-1\right) \exp \left(\bar{t}_{\mathrm{D}}-\bar{t}\right)+1, & \bar{t}>\bar{t}_{\mathrm{D}}\end{cases}
$$

where $\bar{t}_{\mathrm{D}}$ is a normalized electron beam dwell time $\left(\bar{t}_{\mathrm{D}}=t_{\mathrm{D}} / \tau_{\text {out }}\right)$.

Figure 4 shows the normalized adsorbate concentration $\bar{n}=n /\left(s J \tau_{\text {out }}\right)$ versus time at the center of the electron beam for an irradiative depletion of $\tilde{\tau}=1,000$, according to Eq. 1C: the initial adsorbate coverage is 1 at $\bar{t}=0$ and drops to the steady-state coverage of $1 / \tilde{\tau}$ when surface diffusion of adsorbates is neglected.

\section{Growth rate in pulsed FEBID without surface diffusion}

The growth rate (respectively, etch rate) in focused electron beam processing is proportional to the electron flux, adsorbates concentration of adsorbates, volume of the deposited (etched) fragment, and net dissociation cross section:

$R=V n \sigma f$.

The dimensionless form does not depend explicitly on the impinging molecular flux and the volume of the deposited fragment. 
Fig. 4 Evolution of normalized surface coverage of adsorbates at the electron beam center for a given irradiative depletion $\tilde{\tau}=1,000$. When the surface diffusion contribution is neglected, the normalized surface coverage

$\left(\bar{n}=n /\left(s J \tau_{\text {out }}\right)\right)$ at the center of the beam converges to the value of $1 / \tilde{\tau}$. The time evolution is presented on the normalized timescale $\left(\bar{t}=t / \tau_{\text {out }}\right)$.

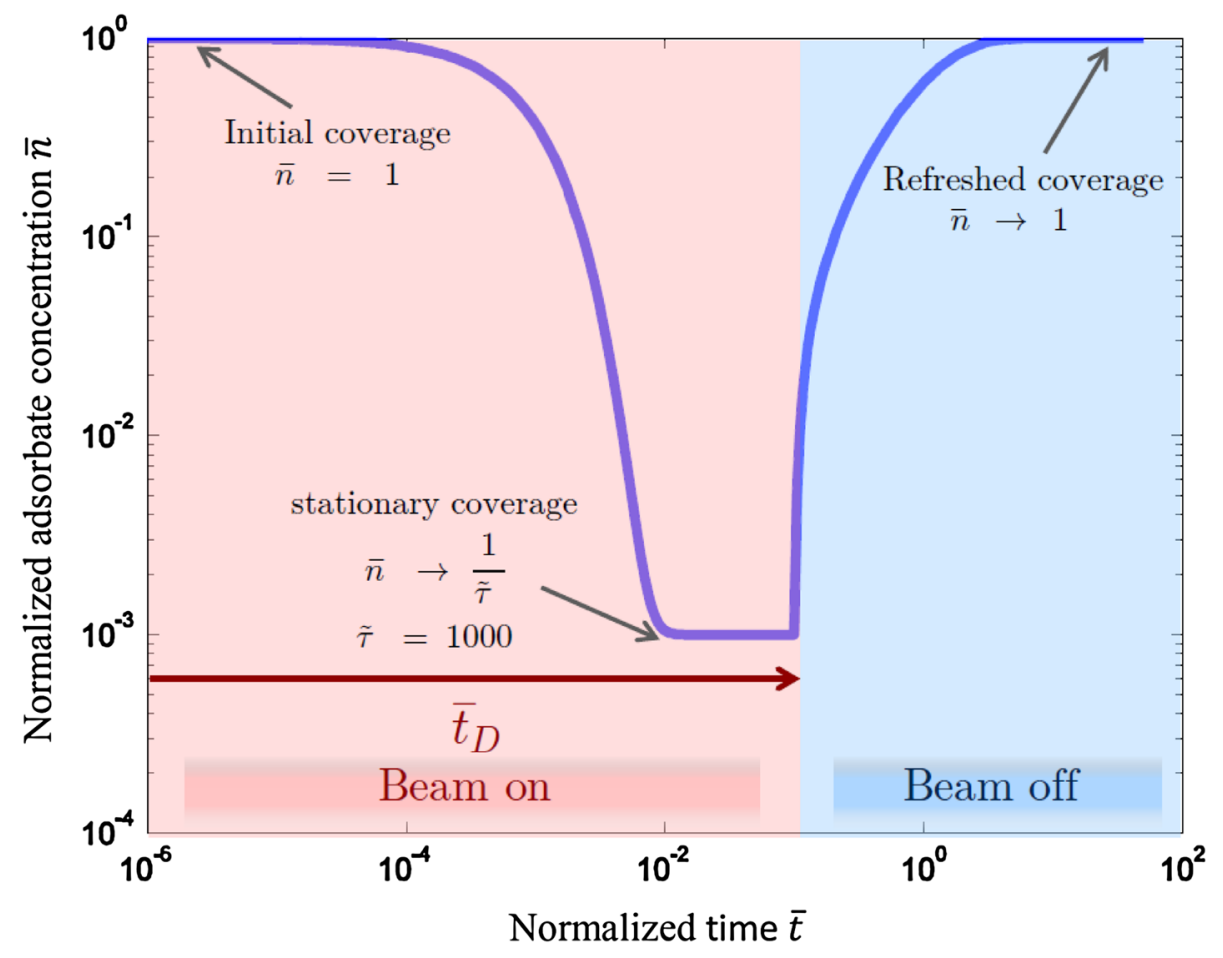

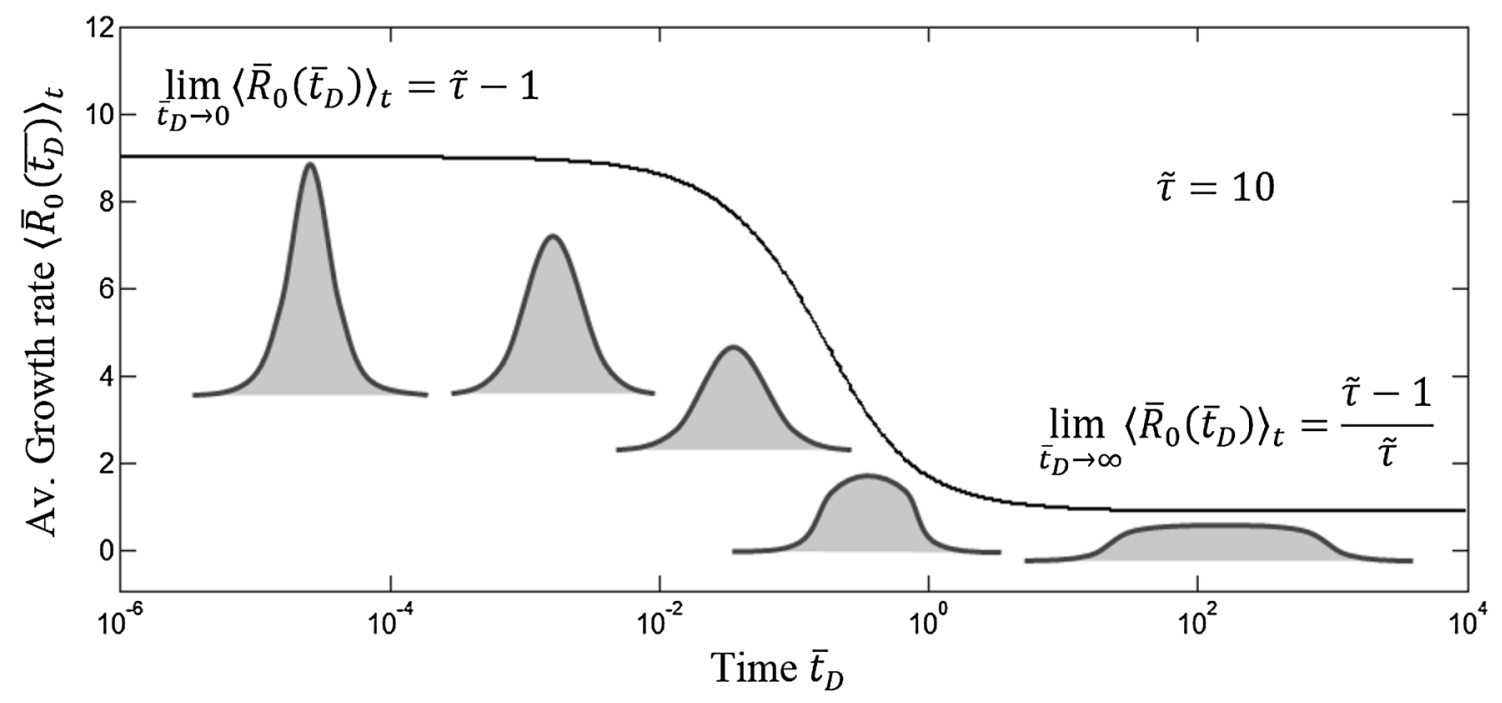

Fig. 5 Dimensionless average growth rate in the center of the electron beam for pulsed FEBID and the parameters $\tilde{\tau}=10, \tilde{\rho}_{\text {out }}=0$. For very short electron beam dwell times, when the surface is still fully replenished by adsorbates $\left(n / n_{\text {out }}=\bar{n}=1\right)$, the dimensionless growth rate is equal to $\tilde{\tau}-1$. The limit of the growth rate at long dwell times in the steady state is equal to $(\tilde{\tau}-1) / \tilde{\tau}$ (neglecting

$\bar{R}=\frac{R}{s J V}=(\tilde{\tau}-1) \bar{f} \bar{n}=(\tilde{\tau}-1) 2^{-\bar{r}^{2}} \bar{n}=(\tilde{k}-1) \bar{n}$.

Neglecting surface diffusion, the average growth (etch) rate for pulsed exposure with an electron beam dwell time $t_{\mathrm{D}}$ $\left(\bar{t}_{\mathrm{D}}=t_{\mathrm{D}} / \tau_{\text {out }}\right)$ is expressed as: surface diffusion). The insets illustrate qualitatively the evolution of the spatial distribution of the average growth rate with exposure time. The evolution of these profiles with dwell time $\bar{t}_{D}$ is described in more details in the further part of this paper also including surface diffusion and exemplified in Fig. 9

$$
\begin{aligned}
& \left\langle\bar{R}\left(\bar{t}_{\mathrm{D}}\right)\right\rangle_{t}=\frac{1}{\bar{t}_{\mathrm{D}}} \int_{0}^{\bar{t}_{\mathrm{D}}} \bar{R}(\bar{t}) \mathrm{d} \bar{t} \stackrel{\tilde{\rho}_{\text {out }} \stackrel{\ll}{\longleftrightarrow}}{\longrightarrow} \\
& \quad \frac{\tilde{k}-1}{\tilde{k}}\left(1+(\tilde{k}-1) \frac{1-\exp \left(-\tilde{k} \bar{t}_{\mathrm{D}}\right)}{\tilde{k} \bar{t}_{\mathrm{D}}}\right)
\end{aligned}
$$


The average growth rate in the center of the beam is obtained by substitution of $\tilde{k}=\tilde{k}(0)=\tilde{\tau}$ in Eq. 5:

$$
\left\langle\bar{R}_{0}\left(\bar{t}_{\mathrm{D}}\right)\right\rangle_{t}=\frac{\tilde{\tau}-1}{\tilde{\tau}}\left(1+(\tilde{\tau}-1) \frac{1-\exp \left(-\tilde{\tau} \bar{t}_{\mathrm{D}}\right)}{\tilde{\tau} \bar{t}_{\mathrm{D}}}\right),
$$

The limits of the deposition (etch) rate for very short and long dwell times of the electron beam in the center of the beam can be expressed in terms of the irradiative depletion parameter:

$$
\begin{aligned}
& \lim _{\bar{t}_{\mathrm{D}} \rightarrow 0}\left\langle\bar{R}_{0}\left(\bar{t}_{\mathrm{D}}\right)\right\rangle_{t}=\tilde{\tau}-1, \\
& \lim _{\bar{t}_{\mathrm{D}} \rightarrow \infty}\left\langle\bar{R}_{0}\left(\bar{t}_{\mathrm{D}}\right)\right\rangle_{t}=\frac{\tilde{\tau}-1}{\tilde{\tau}} .
\end{aligned}
$$

This means that for very short single-spot exposures, the growth rate in the center of the electron beam is a factor $\tilde{\tau}$ larger than for long exposure times $\bar{t}_{D} \gg 1$, see Fig. 5. Furthermore, with regard to the spatial distribution of the growth rate for a single-spot exposure, an increase in the dwell time results in the transformation from a Gaussian toward a flattop average growth rate profile and to the related deposit or etch shape profile.

\section{Lateral resolution parameter}

The lateral resolution parameter describes the lateral size of the obtained deposit $\mathrm{FWHM}_{\mathrm{D}}$ with respect to the size of the electron beam $\mathrm{FWHM}_{\mathrm{B}}$ and is defined as:

$\tilde{\varphi}:=\frac{\mathrm{FWHM}_{\mathrm{D}}}{\mathrm{FWHM}_{\mathrm{B}}}$.

In the electron-limited regime, the deposit shape corresponds to the Gaussian profile of the beam; therefore, it assumes its smallest value which is equal to 1. Generally, the value of the resolution parameter depends on the FEBID regimes illustrated in Fig. 1, and it can be estimated by the scaling laws discussed below.

\section{Scaling laws}

The scaling laws introduced here correlate the lateral deposit (or etch pit) size with the input parameters of the FEBID model. They can be used to recalculate the surface kinetics parameters of FEBID precursor adsorbates [15-17] or to re-adjust exposure parameters to obtain a desired lateral resolution or shape from the process.

1. First scaling law: stationary exposure without surface diffusion

For the stationary-state solution, where no diffusion is present, the lateral resolution parameter is a sole function of irradiative adsorbate depletion $\tilde{\varphi}(\tilde{\tau})$. This scaling law was introduced in [15]. For a Gaussian electron beam profile distribution, it can be expressed as:

$\tilde{\varphi}=\left(\log _{2}(1+\tilde{\tau})\right)^{0.5}$.

2. Second scaling law: stationary exposure with surface diffusion

Including surface diffusion by $\tilde{\rho}_{\text {out }}$ (see Eq. 3) results in a new second scaling law for the lateral resolution given by

$\tilde{\varphi} \cong\left(\log _{2}\left(2+\frac{\tilde{\tau}-1}{1+\tilde{\rho}_{\text {out }}^{2}}\right)\right)^{0.5}$

Figure 6a shows the excellent agreement of Eq. 8 with the values derived from the numerical solution of Eq. 1B, which has been solved using pdepe MATLAB ${ }^{\circledR}$ solver $^{1}$ for onedimensional initial-boundary differential system and using logarithmic transformation of the spatial variable $l=\ln (\bar{r})$, which allows accounting for the adsorbates that are diffusing over long distance far from the irradiated area [21].

Once the surface diffusion of adsorbates becomes strong enough, the resolution parameter is carried to the electronlimited regime with the best lateral resolution. A previously formulated scaling law in [17] is a special version of Eq. 8, derived by substituting the surface diffusion replenishment $\tilde{\rho}_{\text {out }}$ by an analogous parameter, defined at the center of the beam $\tilde{\rho}_{\text {in }}=\tilde{\rho}_{\text {out }} / \sqrt{\tilde{\tau}}$. The version presented in [17] gives the lower limit of the surface diffusion coefficient for a given observed lateral resolution of the deposit (etch pit) and works well for large values of $\tilde{\tau}>20$. The advantage of Eq. 8 is that it can be applied to small values of the depletion parameter and allows to quickly predict how changing the beam setup will influence the lateral resolution, according to Eqs. 2 and 3.

3. Third scaling law: pulsed FEBID without surface diffusion

Neglecting the surface diffusion term, a new third scaling law can be formulated, describing the lateral resolution obtained in the FEBID (or etch) process as a function of irradiative adsorbate depletion $\tilde{\tau}$ and the normalized electron beam dwell time $\bar{t}_{\mathrm{D}}$ (see Eq. 1C):

$\tilde{\varphi} \cong\left(\log _{2}\left(2+\frac{\tilde{\tau}-1}{1+\bar{t}_{\mathrm{D}}^{-1}}\right)\right)^{0.5}$

For the short dwell times, the process is carried out in the electron-limited regime and the resolution parameter is equal to 1, see Fig. 6b. For long dwell times, it corresponds to the values derived from the steady-state solution without

\footnotetext{
${ }^{1}$ http://www.mathworks.com/help/matlab/ref/pdepe.html.
} 
(a)

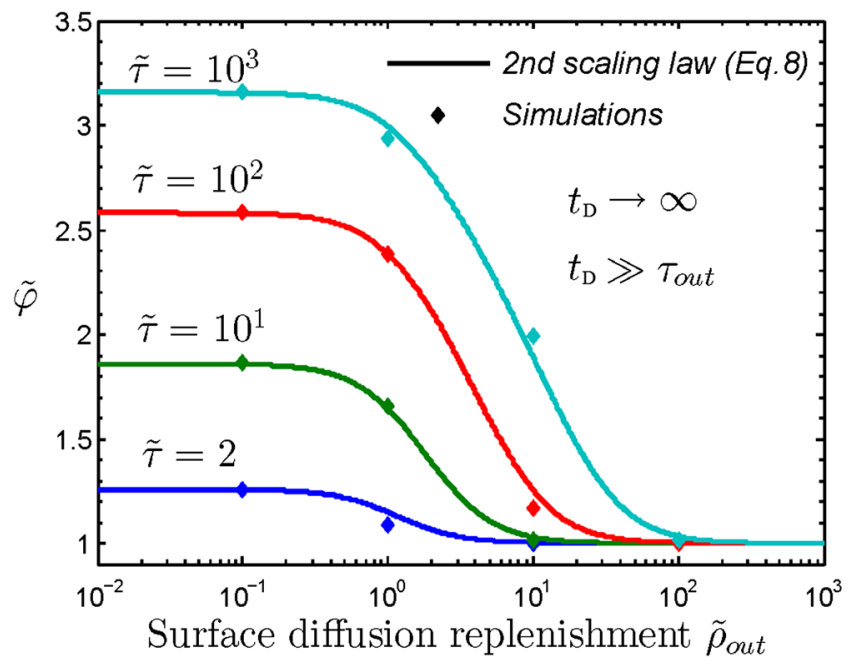

(c)

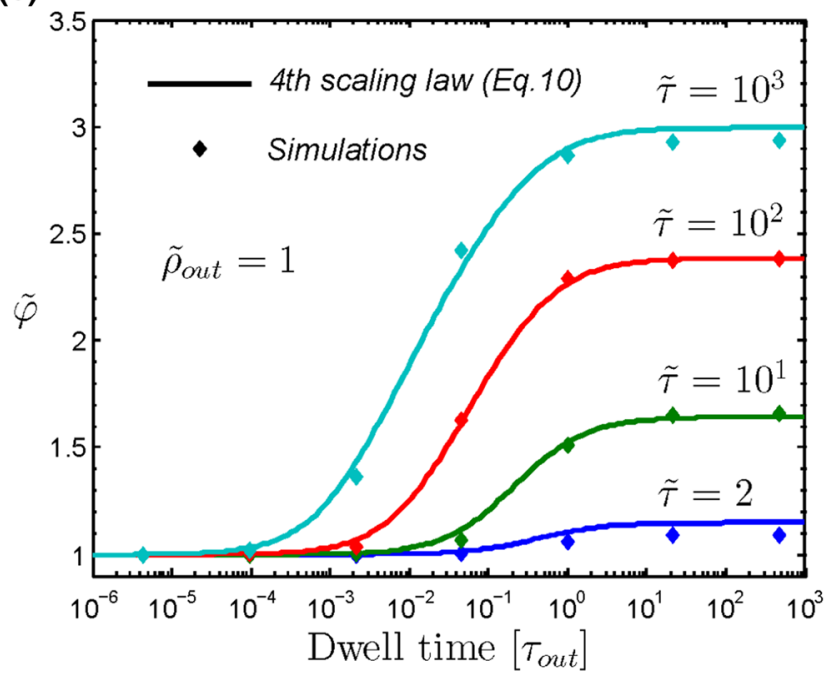

Fig. 6 Illustration of the second, third, and fourth scaling laws for lateral resolution $\tilde{\varphi}$ describing the size of FEB deposits or etch pits which can be obtained by single-spot exposures: a second scaling law: stationary electron beam exposure with surface diffusion-the values for small surface diffusion converge to the first scaling law (Eq. 7); b third scaling law: pulsed electron beam exposure, no surface diffusion-the values corresponding to the long dwell times

surface diffusion which are given by the first scaling law (Eq. 7).

4. Fourth scaling law: pulsed FEBID with surface diffusion

Including the surface diffusion term and taking into account the duration of an electron pulse, a general fourth scaling law can be formulated, describing the lateral resolution obtained in the FEBID (or etch) process as a function of irradiative adsorbate depletion, normalized electron beam dwell time, and surface diffusion replenishment: (b)

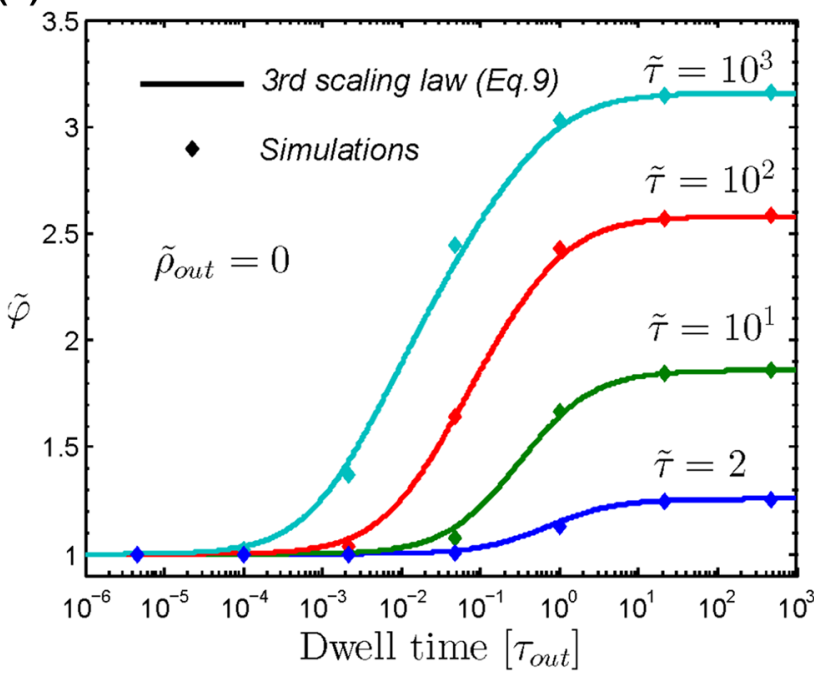

(d)

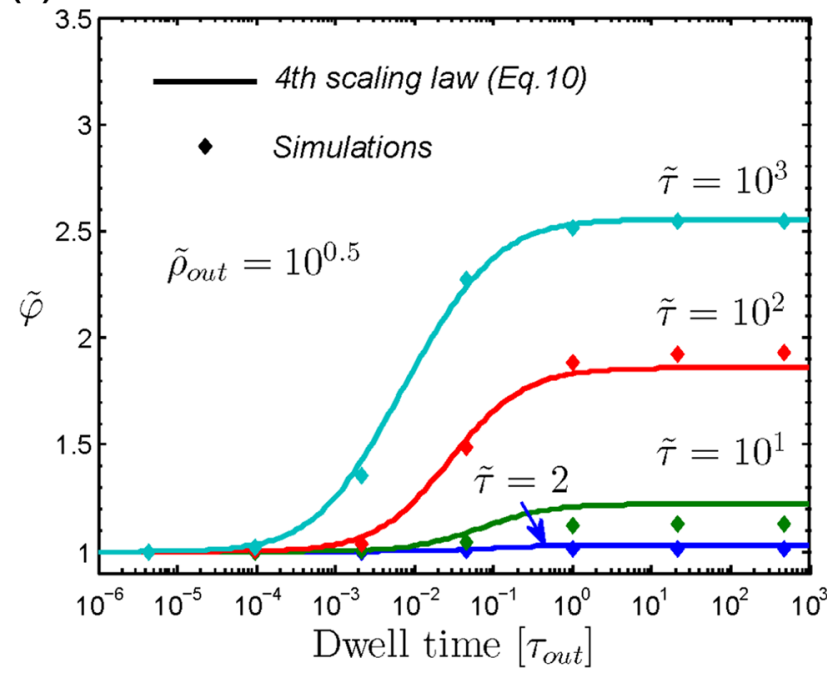

are equal to those given by the first scaling law (Eq. 7); c, $\mathbf{d}$ fourth scaling law: pulsed electron beam exposure with surface diffusionthe deviations from simulations seen at long dwell times correspond to the deviations seen in Fig. 6a. In (b-d), it can be seen that low depletions can accept larger electron beam dwell times before the regime transition from electron-limited to adsorbate-limited or diffusion-enhanced occurs

$\tilde{\varphi} \cong\left(\log _{2}\left(2+\frac{\tilde{\tau}-1}{1+\bar{t}_{\mathrm{D}}^{-1}+\tilde{\rho}_{\text {out }}^{2}}\right)\right)^{0.5}$

Figures $6 \mathrm{c}, \mathrm{d}$ show the comparison of Eq. 10 with the values derived from the numerical solution of Eq. 1B, which has been obtained the analogous way as described in point 2 of the current section. In this case, however, the non-stationary state has been resolved in time.

For the electron-limited (reaction-limited) regime, the resolution parameter is equal to 1 . According to Eq. 10, it can be reached by all the three parameters individually: when the irradiative depletion $\tilde{\tau}$ is close to 1 , when the 

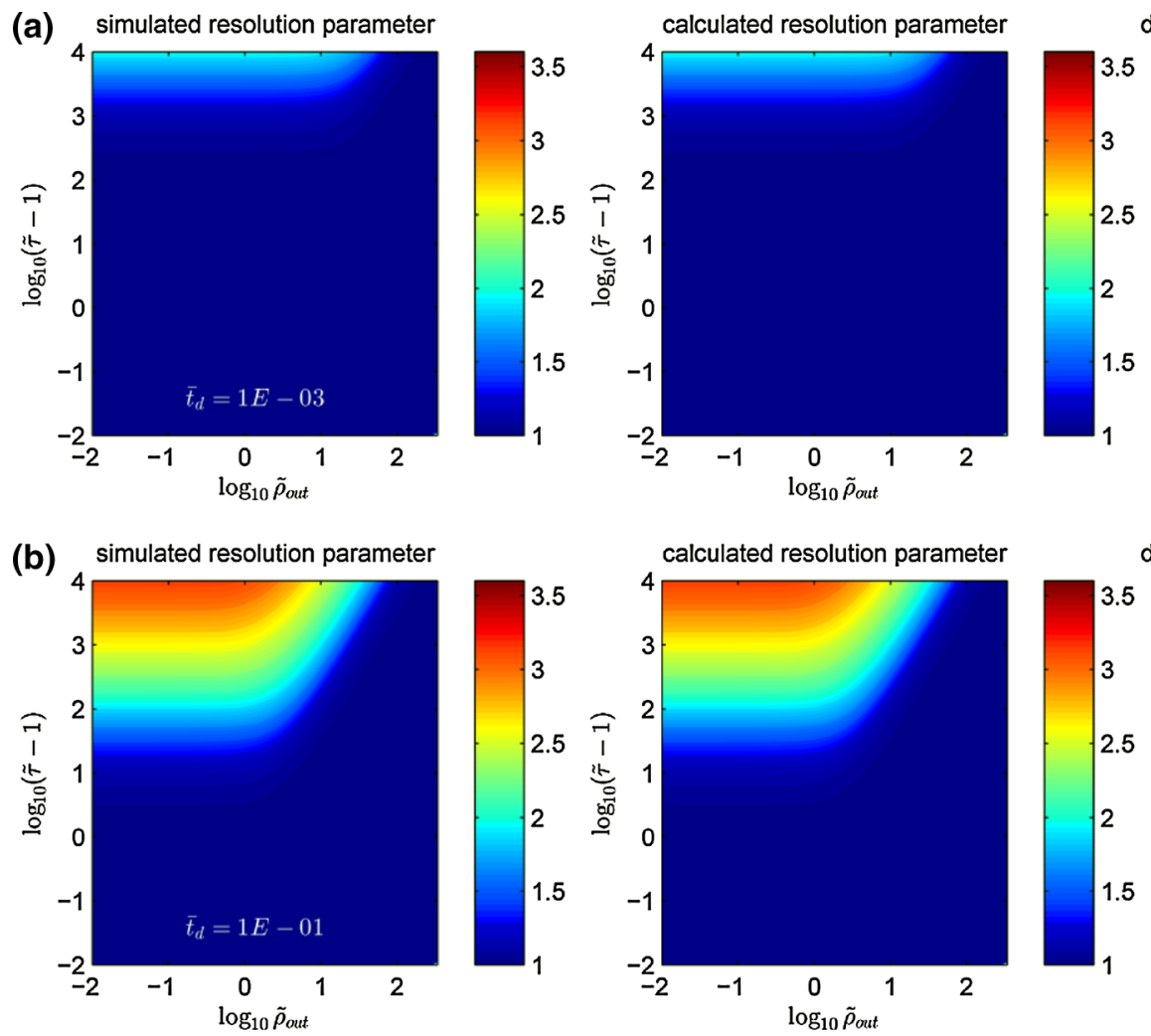

deviation of calculated from simulated in percent
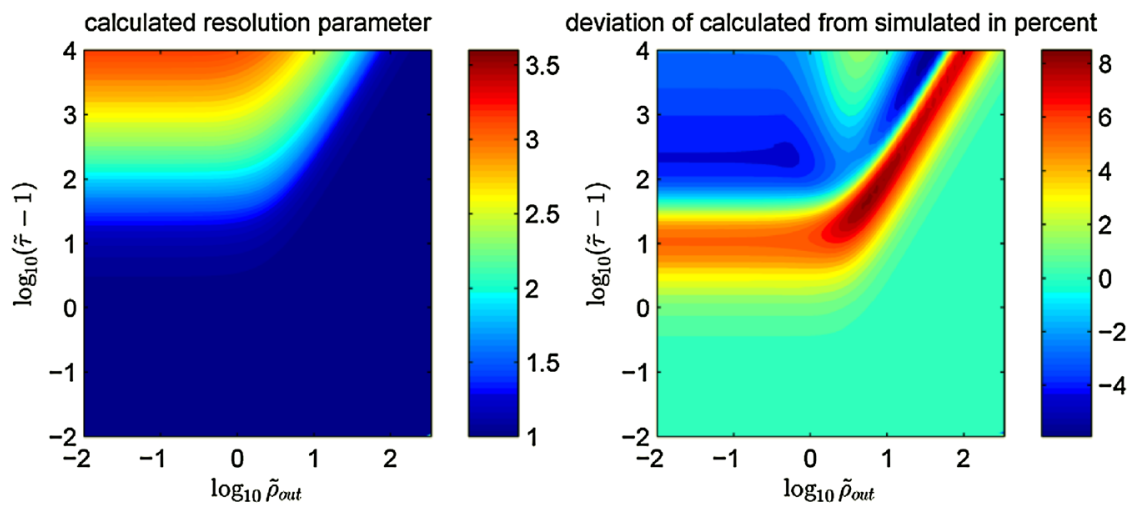

(c) simulated resolution parameter
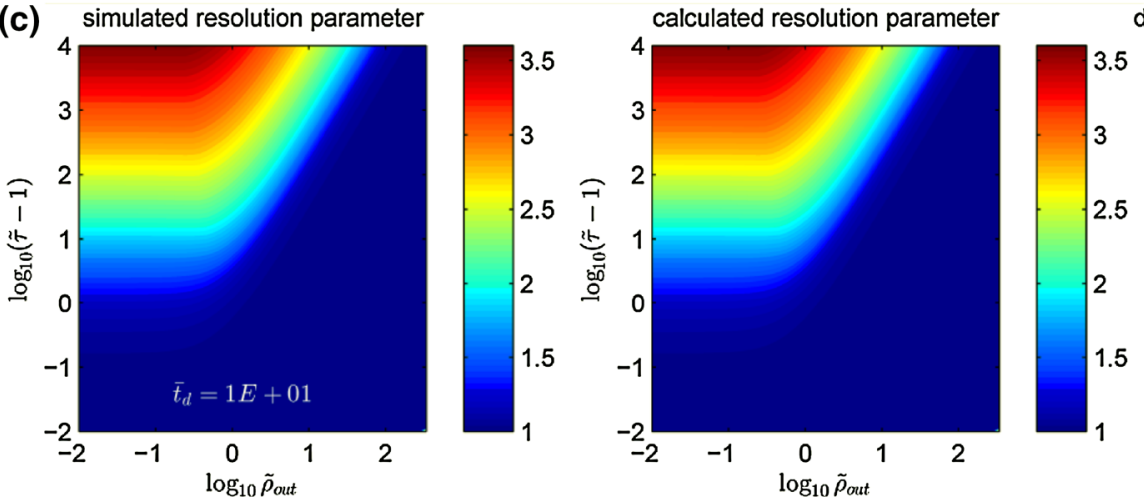

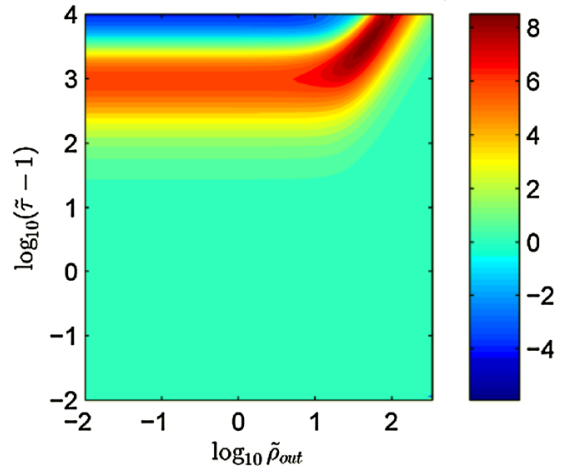

deviation of calculated from simulated in percent

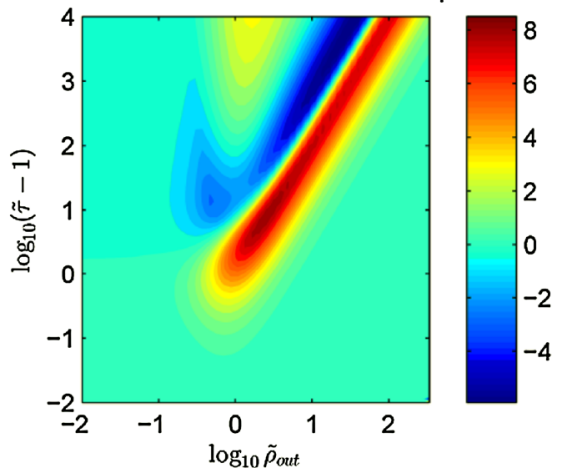

Fig. 7 Evolution of the lateral size $\tilde{\varphi}$ of FEB deposits or etch pits obtained from spot exposures presented in the $\left(\tilde{\tau}, \tilde{\rho}_{\text {out }}\right)$ space for three electron beam exposure dwell times: row $(a) \bar{t}_{D}=10^{-3}$, row $(b)$ $\bar{t}_{D}=10^{-1}$, and row $(c) \bar{t}_{D}=10^{1}$. The left panel shows $\tilde{\varphi}$ as obtained from the numerical solution of (Eq. 1B), the center panel shows $\tilde{\varphi}$ as

surface diffusion replenishment $\tilde{\rho}_{\text {out }}$ is large enough to overcome irradiative depletion, or with a very short electron beam exposure dwell time $\bar{t}_{\mathrm{D}}$ per single spot. In general, the process is carried out in the electron-limited regime when the following statement is true

$\frac{\tilde{\tau}-1}{1+\bar{t}_{\mathrm{D}}^{-1}+\tilde{\rho}_{\text {out }}^{2}} \ll 1$

because then the square root term in Eq. 10 becomes 1. For long electron beam exposure dwell times, the fourth scaling law (Eq. 10) converges to the second scaling law (Eq. 8) obtained from the analytical formula of the fourth scaling law (Eq. 10), and the right panel shows the difference in percent between those two values. The maximum observed deviation in all the cases is below $9 \%$

describing the steady-state solution with surface diffusion. For negligible surface diffusion replenishment, the fourth scaling law reduces to the third scaling law (Eq. 9) describing pulsed FEBID without surface diffusion. For long electron beam dwell times and negligible surface diffusion, it converges to the analytical formula of the first scaling law (Eq. 7) for stationary exposure conditions without surface diffusion.

Figure 7 shows a detailed comparison of the lateral resolution parameter for single-spot exposures obtained by the fourth scaling law (Eq. 10) and by numerically solving 
Fig. 8 Contributions of surface diffusion and adsorbate dissociation at the center of the electron beam (in units of the impinging molecule rate $s J / n_{0}$ ) versus the electron beam exposure dwell time (in units of $\tau_{\text {out }}$. The graphs $(\mathbf{a}-\mathbf{d})$ are presented for selected values of the surface diffusion replenishment parameter $\tilde{\rho}_{\text {out }}$ and irradiative depletion parameter $\tilde{\tau}=2 ; 10 ; 100 ; 1000$. Of note is that the desorption rate scales with the dissociation rate by a factor of $\tilde{\tau}-1$, see Eq. 12 (a)

Surface diffusion rate $\left[\frac{1}{n_{0}} D\left(\frac{\partial^{2} n}{\partial r^{2}}+\frac{1}{r} \frac{\partial n}{\partial r}\right)\right]_{r=0}$

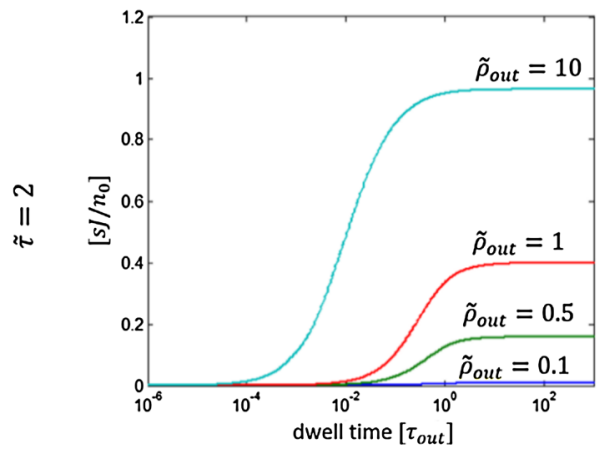

(b)

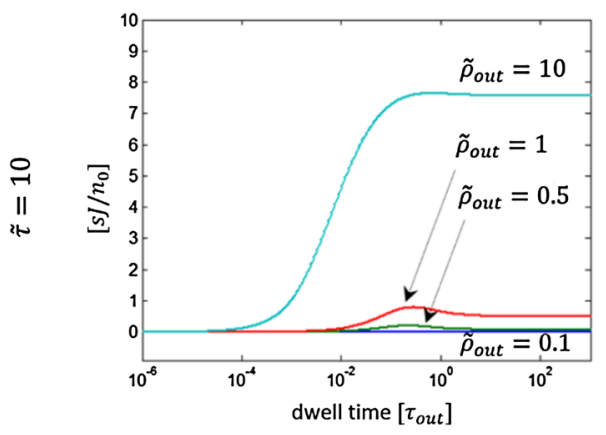

(c)

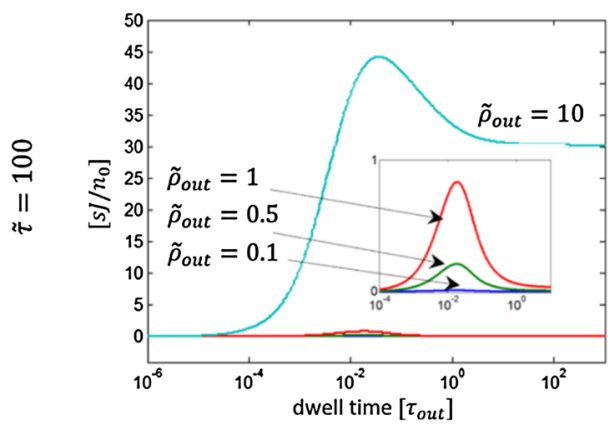

(d)

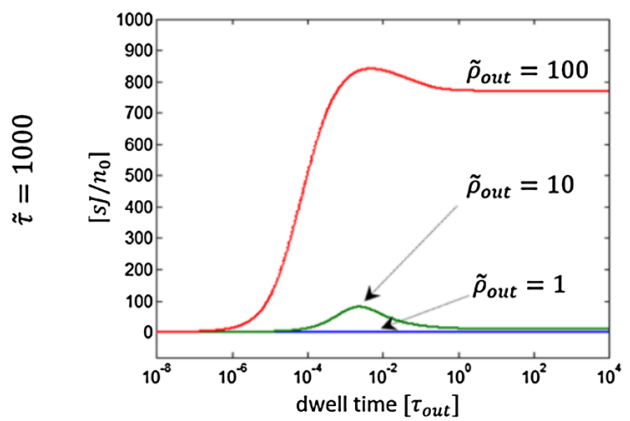

Dissociation rate $\frac{1}{n_{0}} \sigma f_{0} n$

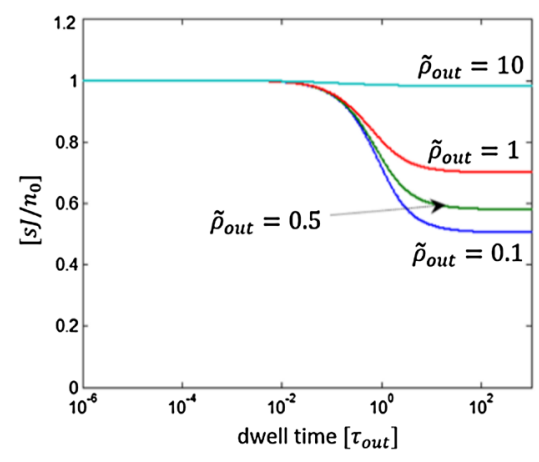

Dissociation rate $\frac{1}{n_{0}} \sigma f_{0} n$

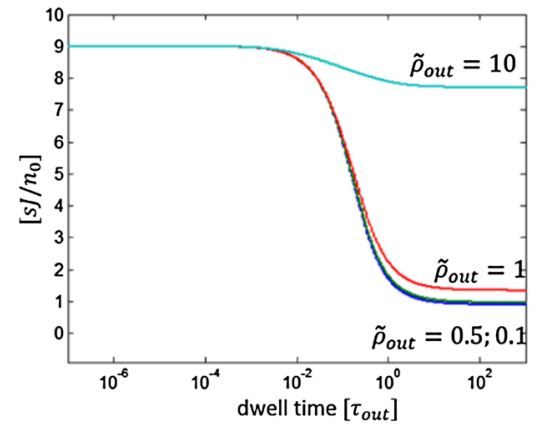

Dissociation rate $\frac{1}{n_{0}} \sigma f_{0} n$

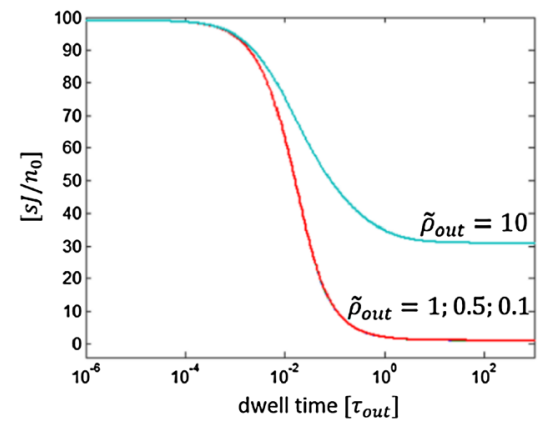

Dissociation rate $\frac{1}{n_{0}} \sigma f_{0} n$

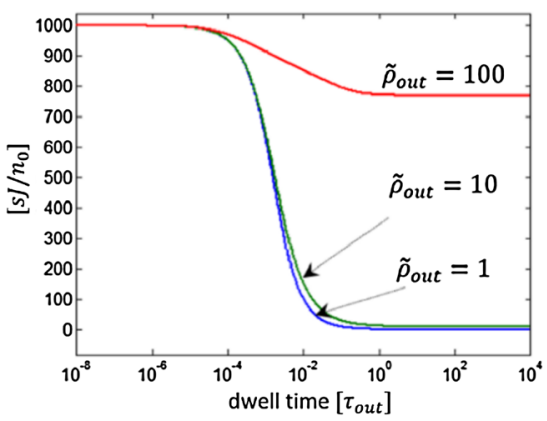




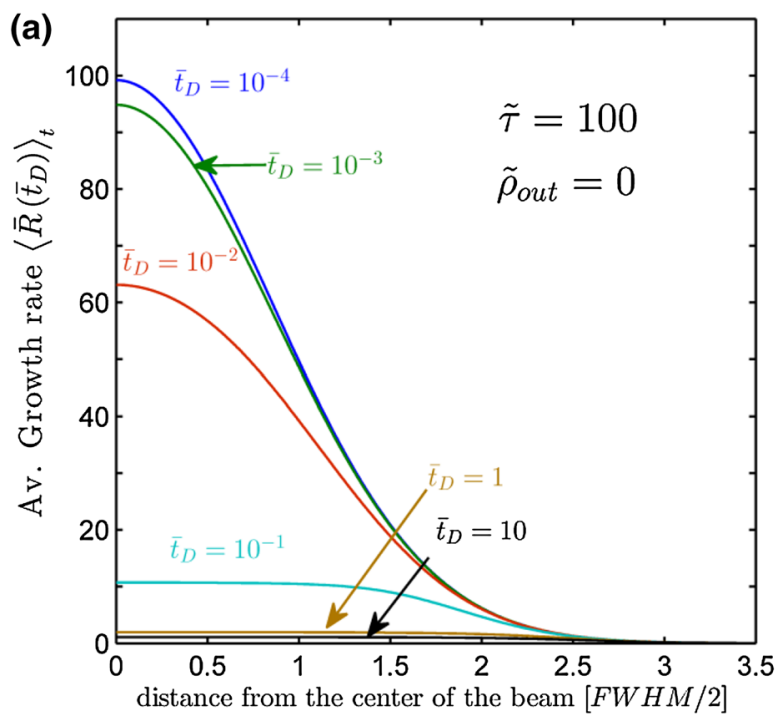

Fig. 9 Average dimensionless growth rates and shapes of (single) spot exposure FEB deposits or etch pits depending on the normalized electron beam exposure dwell time $\bar{t}_{D}$ varying from $10^{-4}$ to $10^{1}$ for an irradiative depletion $\tilde{\tau}=100$. a Negligible effect of surface diffusion $\tilde{\rho}_{\text {out }}=0$ (Eq. 5); b Surface diffusion replenishment $\tilde{\rho}_{\text {out }}=10^{0.5}$, an

the adsorption rate equations (Eqs. 1B and 5) for three fixed electron beam exposure dwell times in the $\left(\tilde{\tau}, \tilde{\rho}_{\text {out }}\right)$ space. The supplementary information contains an animation which covers the full evolution of the lateral size of deposits or etch pits in the range of dwell times $\bar{t}_{\mathrm{D}} \in\left(10^{-6}, 10^{3}\right)$.

\section{Contributions of surface diffusion and adsorbate dissociation in pulsed exposure}

In order to study the contributions of surface diffusion and dissociation at a certain duration of the exposure, we calculated the terms of Eq. 1A at the center of the beam $(r=0)$ for selected values of the $\tilde{\tau}, \tilde{\rho}_{\text {out }}$ input parameter space. The results presented in Fig. 8 (a-d) are given in units of the impingement rate of molecules $\left(s J / n_{0}\right)$ (in units of $\mathrm{s}^{-1}$ ).

The left column panels of Fig. 8 show the diffusion rates $\frac{1}{n_{0}} D\left(\frac{\partial^{2} n}{\partial x^{2}}+\frac{\partial^{2} n}{\partial y^{2}}\right)$ (in units of $\mathrm{s}^{-1}$ ) at the center of the beam for a given value of $\tilde{\tau}$. Only for the small values of depletion $(\tilde{\tau}<10)$, the surface diffusion rate increases monotonously, whereas for the higher depletions a peak appears. The surface diffusion rate at small electron beam dwell times is monotonously rising since the divergence of the adsorbate concentration gradient increases between the inside and outside of the irradiated area due the increasing dwell time, see Fig. 4. The surface diffusion rate plateaus when the steady-state depletion is reached and, for large depletions decays again. This situation is equivalent to the indent shape formation shown in Fig. 9b; due to the

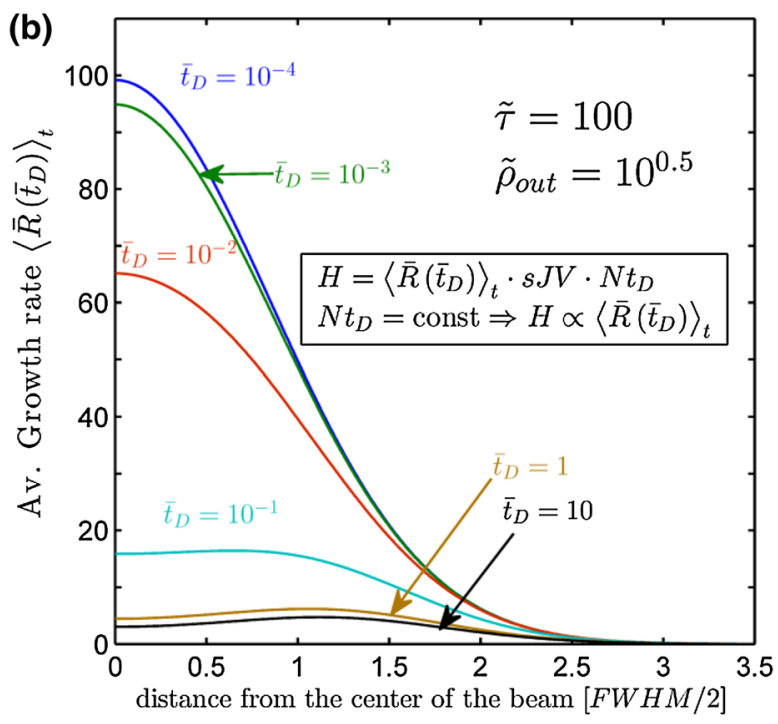

indent shape is obtained by diffusion-enhanced growth rate compared to (a), especially at the rim of the exposed area. Of note is that the profiles can be also obtained for repeated spot exposures on the same pixel, see inset and text

efficient electron-triggered dissociation of surface diffusion adsorbates at the rim of the irradiated area, the center of the deposit is not replenished anymore at longer dwell times. From Fig. 8, it becomes obvious that starting from at a certain dwell time, the diffusive replenishment can become orders of magnitude larger than the replenishment from the gas phase. This was an explanation why FEBID structures fabricated with an intermediate dwell time show a smaller roughness than those with the shortest dwell time values [26]. It can also be an explanation for morphology effects in FEBID structures obtained with different scanning strategies [27] and for the effects of chemical composition changes in two adsorbate systems [28].

In the right column panels of Fig. 8, the adsorbate dissociation rate $\sigma f_{0} n / n_{0}$ (in units of $\mathrm{s}^{-1}$ ) is shown, which is proportional to the growth rate, see Eq. 4 . For the very short dwell times, the growth rate at the center of the beam is constant and equal to $(\tilde{\tau}-1) s J V$, see also Eq. $5 \mathrm{~B}$. It starts to decrease at a given value of the dwell time, which is correlated to the depletion parameter. The limit for the long dwell times depends on the diffusion contribution. For the small values of surface diffusion parameter $\left(\tilde{\rho}_{\text {out }} \ll 0.1\right)$, the stationary growth rate is equal to $(\tilde{\tau}-1) s J V / \tilde{\tau}$, see also Eq. 5C.

The effective desorption rate $\frac{1}{n_{0}} \frac{n}{\tau_{\text {out }}}$ is not explicitly presented in Fig. 8 but scales according to Eq. 2 as:

$\frac{1}{n_{0}} \frac{n}{\tau_{\text {out }}}=\frac{1}{n_{0}} \frac{\sigma f_{0} n}{(\tau-1)}$,

i.e., it is equal to the dissociation rate shown in Fig. 8 divided by the factor $\tilde{\tau}-1$. 


\section{Influence of the electron beam dwell time $\bar{t}_{\mathrm{D}}$ and surface diffusion replenishment $\tilde{\rho}_{\text {out }}$ on the shape of the deposit or etch pit}

In the case of negligible surface diffusion replenishment $\tilde{\rho}_{\text {out }} \ll 0.1$, the analytical formula for the average growth rate $\left\langle\bar{R}\left(\bar{t}_{\mathrm{D}}\right)\right\rangle_{t}$ in Eq. 5 can be used to calculate the shape. The case of surface diffusion replenishment greater than 0.1 necessitates a numerical solution of Eq. 1B for the coverage $\bar{n}(\bar{r}, \bar{t})$ together with a numerical integration of Eq. 5. Figure 9 illustrates the solution for the cases of $\tilde{\rho}_{\text {out }}=0$ and $\tilde{\rho}_{\text {out }}=10^{0.5}$ and for dimensionless dwell times $\bar{t}_{D}$ varying from $10^{-4}$ to $10^{1}$.

The curves in Fig. 9 are generally strictly correct for a single-spot exposure having the boundary and initial conditions stated in Eqs. 1B. The shape of the deposit (thickness) or etch pit (depth) is then simply obtained by multiplying with the electron beam exposure dwell time. Choosing a refresh time long enough for a complete surface replenishment of adsorbates, the shape profiles $H$ for repeated spot exposures (on the same pixel) can be obtained by multiplying by the dwell time $t_{\mathrm{D}}$ and the number of pulse repetitions $N$ :

$H\left(r, t_{\mathrm{D}}\right)=\left\langle R\left(t_{\mathrm{D}}, r\right)\right\rangle_{t} N t_{\mathrm{D}}=\left\langle\bar{R}\left(\bar{t}_{\mathrm{D}}, \bar{r}\right)\right\rangle_{t} \cdot s J V \cdot N t_{\mathrm{D}}$

Keeping the dose constant for all exposures in Fig. 9, i.e., keeping the product of number of repetitions and pulse time $N \cdot t_{D}$ constant, the curves in Fig. 9 would correspond to the exact shape profiles obtained at a given dose. Figure 9 is also an illustration of how the growth rate evolves during a spot exposure: it is Gaussian at very low electron beam dwell times and undergoes all profiles from rounded, flattop (negligible surface diffusion), to indent. These profiles relate to different regimes as shown in Fig. 1. According to the dwell time chosen in an exposure experiment, the FEB growth or etching will thus proceed in various regimes. The consequence even for single-spot deposits is that the bottom layer could be deposited in the electron-limited regime while the top layer parts would be deposited in the adsorbate-limited/diffusion-enhanced regime, i.e., the resulting deposit material would be inhomogeneous with thickness, which is especially important for electrical contacts of FEBID material with electrodes.

\section{Conclusions}

In this work, we presented a new approach to the continuum model for pulsed FEBID, with a set of parameters $\left\{\tilde{\tau}, \tilde{\rho}_{\text {out }}\right\}$, describing independently the surface diffusion and the dissociation contributions, which couple the surface kinetics parameters of adsorbates with the electron beam settings. The growth rate for pulsed FEBID is expressed as function of the adsorbate depletion parameter with the respective limits for short and long dwell times. The application of dimensionless parameters allows us to predict how changes in the electron beam setup will influence the process and how it will determine the deposit shape. Three new analytical scaling laws governing the lateral size of deposits or etch pits in gas-assisted focused electron beam-induced processing were formulated as function of the adsorbate surface diffusion term, the electron beam dwell time for a single-spot exposure, and the irradiative adsorbate depletion. The analytical scaling laws were mapped against the exact simulation results and show very good agreement. The maximum mapped error in the variable space was about $8 \%$. The evolution of the contributions of surface diffusion and electron-induced adsorbate dissociation as a function of electron beam dwell (exposure) time was calculated in detail from the numerical solution, showing a peak of the surface diffusive replenishment at the center of the irradiated area at a certain exposure time being significantly higher than the replenishment by the impinging molecular precursor flux from the gas phase. The decay of surface diffusion replenishment at the center for short and long exposure dwell times is due to the low divergence of the adsorbate profile and the dissociation of adsorbates at the rim of the irradiated area, respectively, and results in specific deposit shapes and eventually material along the period of exposure.

Acknowledgments The authors would like to acknowledge the contribution of the COST Action MP0901.

Open Access This article is distributed under the terms of the Creative Commons Attribution License which permits any use, distribution, and reproduction in any medium, provided the original author(s) and the source are credited.

\section{References}

1. I. Utke, A. Gölzhäuser, Angew. Chem. Int. Ed. 49 (2010). doi:10. 1002/anie.201002677

2. J.M. De Teresa, A. Fernández-Pacheco, R. Códoba, J. Sesé, M.R. Ibarra, I. Guillamón, H. Suderow, S. Vieira, MRS proc. CC04-09, $1880(2009)$

3. A. Fernández-Pacheco, J.M. De Teresa, A. Szkudlarek, R. Córdoba, M.R. Ibarra, D. Petit, L. O'Brien, H.T. Zeng, E.R. Lewis, D.E. Read, R.P. Cowburn, Nanotechnology (2009). doi:10.1088/ 0957-4484/20/47/475704

4. M. Gavagnin, H.D. Wanzenboeck, D. Belic, E. Bertagnolli, ACS Nanotechnol. (2012). doi:10.1021/nn305079a

5. T. Brintlinger, M.S. Fuhrer, J. Melngailis, I. Utke, T. Bret, A. Perentes, P. Hoffmann, M. Abourida, P. Doppelt, J. Vac. Sci. Technol. B 23, 3174 (2005). doi:10.1116/1.2130355

6. S. Kim, D. Kulkarni, K. Rykaczewski, M. Henry, V.V. Tsukruk, A. Fedorov, IEEE Trans. Nanotechnol. 11(6), 1223-1230 (2012). doi:10.1109/TNANO.2012.2220377 
7. ChH Schwalb, Ch. Grimm, M. Baranowski, R. Sachser, F. Porrati, H. Reith, P. Das, J. Müller, F. Völklein, A. Kaya, M. Huth, Sensors (2010). doi:10.3390/s101109847

8. M. Gabureac, L. Bernau, I. Utke, G. Boero, Nanotechnology (2010). doi:10.1088/0957-4484/21/11/115503

9. F. Kolb, K. Schmoltner, M. Huth, A. Hohenau, J. Krenn, A. Klug, E.J. List, H. Plank, Nanotechnology (2013). doi:10.1088/09574484/24/30/305501

10. K. Höflich, R.B. Yang, A. Berger, G. Leuchs, S. Christiansen, Adv. Mater. 23. (2011). doi:10.1002/adma.201004114

11. I. Utke, M.G. Jenke, Ch. Röling, P.H. Thiesen, V. Iakovlev, A. Sirbu, A. Mereuta, A. Calimanc, E. Kaponc, Nanoscale (2011). doi:10.1039/C1NR10047E

12. A. Perentes, P. Hoffmann, J. Vac. Sci. Technol. B 25, 2233 (2007). doi:10.1116/1.2798746

13. H.D. Wanzenboeck, M. Fischer, R. Svagera, J. Wernisch, E. Bertagnolli, J. Vac. Sci. Technol. B 24, 2755 (2006). doi:10.1116/ 1.2393245

14. I. Utke, P. Hoffmann, J. Melngailis, J. Vac. Sci. Technol. B 26, 4 (2008). doi:10.1116/1.2955728

15. I. Utke, V. Friedli, M. Purrucker, J. Michler, J. Vac. Sci. Technol. B 25, 6 (2007). doi:10.1116/1.2789441

16. A. Szkudlarek, M. Gabureac, I. Utke, J. Nanosci. Nanotechnol. 11, 9 (2011). doi:10.1166/jnn.2011.5066

17. J.D. Fowlkes, P.D. Rack, ACS Nano (2010). doi:10.1021/ nn901363a
18. M.G. Lassiter, P.D. Rack, Nanotechnology (2008). doi:10.1088/ 0957-4484/19/45/455306

19. S. Randolph, M. Toth, J. Cullen, C. Chandler, Ch. Lobo, Appl. Phys. Lett. 99, 213103 (2011). doi:10.1063/1.3662928

20. M. Toth, Ch.J. Lobo, G. Hartigan, W.R. Knowles, J. Appl. Phys. 101, 054309 (2007). doi:10.1063/1.3662928

21. Ch.J. Lobo, M. Toth, R. Wagner, B.L. Thiel, M. Lysaght, Nanotechnology (2008). doi:10.1088/0957-4484/19/02/025303

22. L. Bernau, M. Gabureac, R. Erni, I. Utke, Angew. Chem. Int. Ed. 49. (2010). doi:10.1002/anie.201004220

23. M. Huth, F. Porrati, Ch. Schwalb, M. Winhold, R. Sachser, M. Dukic, J. Adams, G. Fantner, Beilstein J. Nanotechnol. (2012). doi:10.3762/bjnano.3.70

24. J. Bishop, Ch.J. Lobo, A. Martin, M. Ford, M. Phillips, M. Toth, Phys. Rev. Lett. 109, 146103 (2012). doi:10.1103/PhysRevLett. 109.146103

25. W.F. van Dorp, Th.W. Hansen, J.B. Wagner, J.T.M. De Hosson, Beilstein J. Nanotechnol. (2013). doi:10.3762/bjnano.4.56

26. R. Winkler, J. Fowlkes, A. Szkudlarek, I. Utke, PhD Rack, H. Plank, ACS Appl. Mater. Interfaces (2014). doi:10.1021/ am405591d

27. A. Szkudlarek, M. Gabureac, I. Utke, ECS Trans. 50. (2013). doi:10.1149/05012.0495ecst

28. M. Gabureac, L. Bernau, I. Utke, J. Nanosci. Nanotechnol. 11, 9 (2011). doi:10.1166/jnn.2011.5067 